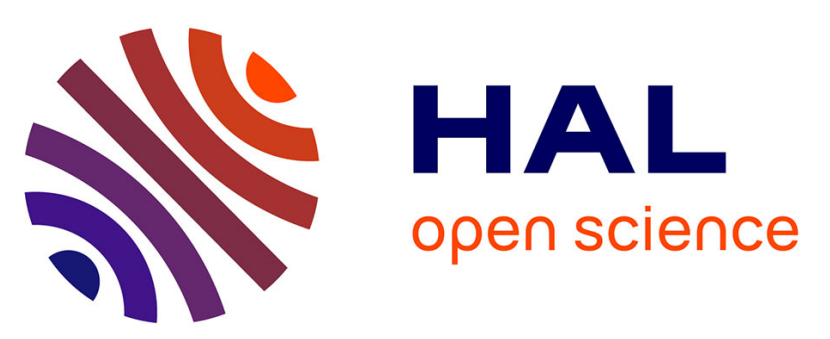

\title{
Generalized Array Factor Approach to the Assessment of Discrete Tapered Nonuniform Leaky-Wave Antenna
}

Olivier Rance, Pierre Lemaitre-Auger, Romain Siragusa, Etienne Perret

\section{To cite this version:}

Olivier Rance, Pierre Lemaitre-Auger, Romain Siragusa, Etienne Perret. Generalized Array Factor Approach to the Assessment of Discrete Tapered Nonuniform Leaky-Wave Antenna. IEEE Transactions on Antennas and Propagation, 2015, 63 (9), pp.3868-3877. 10.1109/TAP.2015.2444440 . hal02066635

\section{HAL Id: hal-02066635 \\ https://hal.science/hal-02066635}

Submitted on 12 May 2020

HAL is a multi-disciplinary open access archive for the deposit and dissemination of scientific research documents, whether they are published or not. The documents may come from teaching and research institutions in France or abroad, or from public or private research centers.
L'archive ouverte pluridisciplinaire HAL, est destinée au dépôt et à la diffusion de documents scientifiques de niveau recherche, publiés ou non, émanant des établissements d'enseignement et de recherche français ou étrangers, des laboratoires publics ou privés. 


\title{
Generalized Array Factor Approach to the Assessment of Discrete Tapered Non-uniform Leaky Wave Antenna
}

\author{
O. Rance, P. Lemaître-Auger, R. Siragusa, E. Perret, Senior Member IEEE
}

\begin{abstract}
A generalization of the array factor approach of a nonuniform leaky wave antenna (LWA) is proposed for the modelling of discrete tapered LWAs. The demonstration is based on the Fourier transform of the illumination amplitude of a LWA and is thus quite general. This analysis approach can be helpful for design. When it is used in combination with classical taperization techniques, it provides an easy and powerful conception tool. The validity of the method is demonstrated with the Holey and the Honey LWAs. Taylor and cosine distributions were simulated and sidelobe level reduction up to $-40 \mathrm{~dB}$ were obtained. A Holey antenna designed to work at $1.7 \mathrm{GHz}$ with a cosine distribution was realized. Its radiation pattern was measured and it shows an acceptable agreement with theory and numerical simulations.
\end{abstract}

Index Terms - non-uniform leaky-wave antenna, discrete antenna, array factor, sidelobe level minimization, Holey antenna, Honey antenna.

\section{INTRODUCTION}

$\mathrm{O}$ NE-DIMENSIONAL LWAs are capable to produce high directivity fan beams with a simple feed compared to antenna arrays, and they exist in a planar form nowadays. Furthermore, in the last decade, the difficulty of emitting at broadside was overcome for all kinds of LWAs [1]. All those very interesting properties are making LWAs attractive for a lot of applications.

Space-wave LWAs belong to the general class of traveling wave antennas. The radiating wave on a LWA is a fast wave, which leaks power all along the length of the waveguide. Onedimensional LWAs are classified as uniform or periodic depending on the radiation mechanism they use $[2,3]$. In the first category, the guiding structure is uniform all along the propagation axis and supports a leaky wave. The phase constant of the leaky mode, $\beta$, is smaller than the wavenumber in vacuum, $k_{0}: \beta<k_{0}$. In the second category, the unperturbed structure supports a non-radiating wave with $: \beta>k_{0}$. A periodical perturbation of period $p$ is introduced causing the apparition of space harmonics. Radiation is caused by at least one of those space harmonics for which $\beta_{n .}=\beta+2 \pi n / p<k_{0}$ [4]. According to this definition, a structure showing a periodicity in its geometry is not necessarily classified as a

O. Rance, P. Lemaître-Auger, R. Siragusa, E. Perret are with the Laboratoire de Conception et d'Intégration des Systèmes (LCIS) Grenoble Institute of Technology, Valence, France (e-mail: olivier.rance@lcis.grenoble-inp.fr; pierre.lemaitre-auger@lcis.grenobleinp.fr_romain.siragusa@lcis.grenoble-inp.fr; etienne.perret@lcis.grenoble-inp.fr). E. Perret is also with the Institut

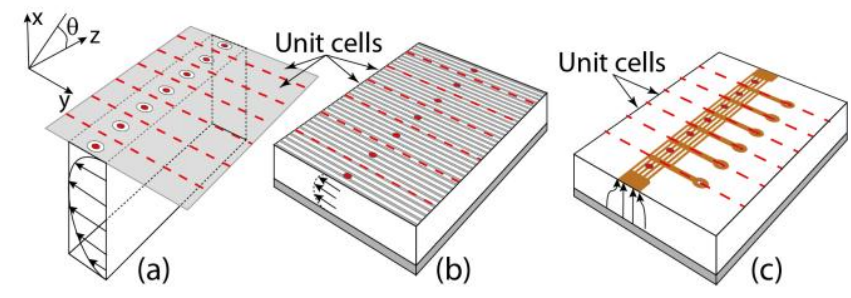

Fig. 1 Overview of different quasi-uniform LWAs. (a) Holey LWA consisting in a rectangular waveguide guiding the $\mathrm{TE}_{10}$ mode over which holes are drilled [6]. (b) Honey LWA consisting in a dielectric waveguide guiding the $\mathrm{TE}_{10}$ mode over which an array of metal strips are put [5]. (c) CRLH LWA whose unit cell is made of interdigital capacitors and stub ended by a via [3].

periodic LWA. It depends on the radiating mode.

Quasi-uniform LWAs are a subset of the uniform-LWA category. However, they slightly differ from uniform LWAs because of the presence of a periodic structure, whose period $p$ is small compared to the guided wavelength, $\lambda_{g}: p / \lambda_{g} \rightarrow 0$. Due to the small periodicity, space harmonics do not play any role in the radiation. The radiation comes from the perturbed fundamental mode which is fast. Thus quasi-uniform LWAs show an electrical behavior similar to that of uniform LWAs [1-3]. For some structures, like composite right/left handed (CRLH) LWA [3] or Honey LWA [5], the periodic structure is the waveguide itself. For other structures like the Holey LWA [6], the periodic structure slightly perturbs the guided mode transforming it into a leaky mode. A schematic representation of those antennas is given in Fig. 1. The classification of the LWAs includes tapered structures even if they are neither strictly uniform nor periodic [2].

The fact that quasi-uniform LWAs have a small periodic structure is of special interest because the emitters can be assimilated to point emitters located at the center of the corresponding unit cell (UC) (Fig. 1). Caloz et al. took advantage of that and proposed the array factor approach to compute the radiation patterns of non-tapered CRLH LWAs [7]. A generalization of the approach is proposed in this paper in order to describe general discrete LWAs by an equivalent array factor. This allows applying classical array results to the domain of LWAs. With the new formulation, the radiation pattern of structures for which the aperture can be tapered is easily described.

The treatment of tapered discrete LWA has already been addressed in literature with discrete approach [8,9] but no closed-form formula of the radiation pattern has been derived until now. 
The generalization of the array factor approach is presented in sect. II. In sect. III, a design procedure for discrete nonuniform LWA is proposed. The proposed method is illustrated with numerical examples in sect. IV. A third example of a tapered quasi-uniform LWA was realized and measured. Results are presented and compared with the theory and numerical simulations in sect. $\mathrm{V}$.

\section{GENERALIZED ARRAY FACTOR APPROACH}

The physical principle of the array factor approach as it was proposed in [7] is based on the fact that the emitters of quasiuniform LWAs can be assimilated to localized sources. So, following that idea, the radiation pattern of non-tapered quasiuniform LWA is obtained by the product of the array factor (AF) with the radiation pattern of a single emitter. Because of the condition that $p / \lambda_{g} \rightarrow 0$, in [7], the emitters were considered point sources and their radiation pattern isotropic, so the radiation pattern of a LWA was equal to the AF.

In the case of tapered non-uniform LWAs, the attenuation constant, $\alpha$, is not constant and the formulas in [7] can no longer be used directly. We show here that a more general approach of the problem leads to the formulation of a generalized array factor approach (GAFA) for discrete tapered non-uniform LWAs.

\section{A. Derivation of the Array Factor Formula}

The radiation pattern in the far-field, $R(\theta)$, is the Fourier transform of the aperture illumination. For a continuous LWA oriented along the $z$-axis, it is [2]:

$$
R(\theta)=\int_{0}^{L} \widetilde{M}(z) e^{j k_{0} z \sin \theta} d z,
$$

where $L$ is the length of the LWA, $k_{0}$ is the wave number in vacuum and $\theta$ is the observation angle (i.e. the elevation angle, see Fig. 1). $\widetilde{M}(z)$ denotes the continuous complex aperture illumination producing the desired radiation pattern. For continuous LWAs, $\widetilde{M}(z)$ is function of the attenuation constant, $\alpha(z)$, and of the phase constant in the waveguide, $\beta(z)[2,8]$ :

$$
\widetilde{M}(z)=A \sqrt{\alpha(z)} e^{-\int_{0}^{z} \alpha(\tau) d \tau} e^{-j \int_{0}^{z} \beta(\tau) d \tau},
$$

where $A$ is a proportionality constant.

Discrete non-uniform LWAs are divided into $N$ linear segments corresponding geometrically to the unit cells, $S_{n}$ :

$$
S_{n}=\left[z_{n} ; z_{n}+d_{n}\right],
$$

where $z_{n}$ is the position of the $n^{\text {th }} \mathrm{UC}$ along the $z$-axis, and $d_{n}$ is its length. The average values of the attenuation and phase constants along a UC are denoted by $\alpha_{\mathrm{n}}$ and $\beta_{\mathrm{n}}$. To obtain a specific radiation pattern with a discrete non-uniform LWA, $\alpha_{\mathrm{n}}$ and $\beta_{\mathrm{n}}$ will be chosen in such a way that they follow a continuous tapering distribution known to give the desired radiation pattern [10]:

$$
\alpha_{n}=\alpha\left(z_{n}\right), \quad \beta_{n}=\beta\left(z_{n}\right),
$$

The attenuation and phase constants along the discrete structure (here denoted with the subscript $s$ ) are assumed to be described by:

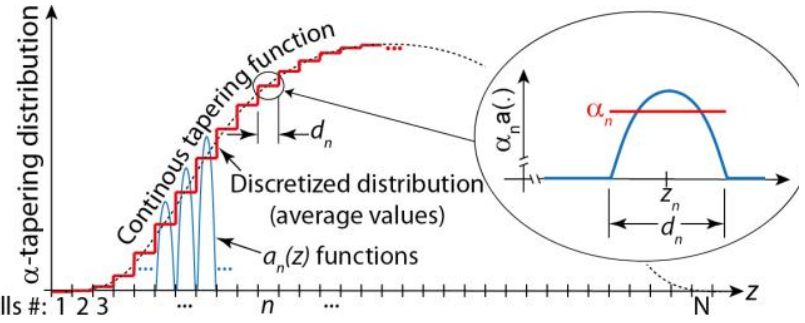

Unit cells \#: $1233 \quad \ldots \quad n \quad \ldots$
Fig. 2 Schematic representation of the model of the attenuation constant for a discrete non-uniform LWA.

$\alpha_{s}(z)=\sum_{n=1}^{N} \alpha_{n} a\left(\frac{z-z_{n}}{d_{n}}\right), \quad \beta_{s}(z)=\sum_{n=1}^{N} \beta_{n} b\left(\frac{z-z_{n}}{d_{n}}\right)$,

where the normalized functions, $a(z)$ and $b(z)$, represent the distribution of the attenuation and phase constants along a unit cell:

$$
\int_{0}^{1} a(z) d z=1, \quad \int_{0}^{1} b(z) d z=1,
$$

$a(z)$ and $b(z)$ are usually unknown inside the integration bounds of (6) but are equal to zero outside of it. A schematic illustration of a distribution of $\alpha_{n}, \alpha_{s}(z)$ and also of a function $a(z)$ are given in Fig. 2.

For discrete non-uniform LWA, equation (1) thus becomes:

$$
R_{S}(\theta)=\sum_{n=1}^{N} \int_{z_{n}}^{z_{n}+d_{n}} \widetilde{M}_{n}(z) e^{j k_{0} z \sin \theta} d z,
$$

with:

$$
\begin{aligned}
& \widetilde{M}_{n}(z)=A \sqrt{\alpha_{n} a\left(\frac{z-z_{n}}{d_{n}}\right)} \\
& \times \exp \left(-\sum_{i=1}^{n-1} \alpha_{i} \int_{S_{i}} a\left(\frac{\tau-z_{i}}{d_{i}}\right) d \tau-\alpha_{n} \int_{z_{n}}^{z} a\left(\frac{\tau-z_{n}}{d_{n}}\right) d \tau\right) \\
& \times \exp \left(-j \sum_{i=1}^{n-1} \beta_{i} \int_{S_{i}} b\left(\frac{\tau-z_{i}}{d_{i}}\right) d \tau-j \beta_{n} \int_{z_{n}}^{z} b\left(\frac{\tau-z_{i}}{d_{i}}\right) d \tau\right) .
\end{aligned}
$$

The radiation pattern of a discrete non-uniform LWA immediately follows:

$$
\begin{gathered}
R_{S}(\theta)=A \sum_{n=1}^{N} \sqrt{\alpha_{n}} \exp \left[-\sum_{i=1}^{n-1}\left(\alpha_{i}+j \beta_{i}-j k_{0} \sin \theta\right) d_{i}\right] \\
\times \int_{S_{n}} \sqrt{a\left(\frac{z-z_{n}}{d_{n}}\right)} \exp \left[-\alpha_{n} \int_{z_{n}}^{z} a\left(\frac{\tau-z_{n}}{d_{n}}\right) d \tau\right] \\
. \exp \left[-j \beta_{n} \int_{z_{n}}^{z} b\left(\frac{\tau-z_{n}}{d_{n}}\right) d \tau\right] e^{j k_{0} \sin \theta\left(z-z_{n}\right)} d z .
\end{gathered}
$$

A change of variable: $t=\left(z-z_{n}\right) / d_{n}$, is done in the integral over a UC in order to simplify (9):

$R_{S}(\theta)=A \sum_{n=1}^{N} d_{n} \sqrt{\alpha_{n}} \mathrm{e}^{-\sum_{i=1}^{n-1}\left(\alpha_{i}+j \beta_{i}-j k_{0} \sin \theta\right) d_{i}} \times S_{n}(\theta)$,

with: 


$$
\begin{aligned}
& S_{n}(\theta)=\int_{0}^{1} \sqrt{a(t)} \exp \left(-\alpha_{n} d_{n} \int_{0}^{t} a(\tau) d \tau\right) \\
& \times \exp \left(-j \beta_{n} d_{n} \int_{0}^{t} b(\tau) d \tau\right) e^{j k_{0} \sin \theta t d_{n}} d t .
\end{aligned}
$$

The integral over a UC, (11), represents the normalized radiation pattern of a single UC. Formally, it is different for each UC. However, it is reasonable to presume that the differences from a UC to another are negligibly small. Indeed, in a non-uniform LWA, the physical structure of a UC is the same for all UCs (only the dimensions change from a UC to the other) and the variations of $\alpha_{n}, \beta_{n}$, and $d_{n}$ are small. So, with that assumption, the integrals of (11) are assumed to be all equal to the same function of the angle $\theta$, here so called $S(\theta)$. Physically, this quantity is the radiation pattern of a single UC. Finally, by identifying the magnitude and phase terms for each UC in (10):

$$
\begin{gathered}
A_{n}=A d_{n} \sqrt{\alpha_{n}} \exp \left(-\sum_{i=1}^{n-1} \alpha_{i} d_{i}\right), \\
\xi_{n}=\sum_{i=1}^{n-1} \beta_{i} d_{i},
\end{gathered}
$$

we obtain the expression:

$$
R_{S}(\theta)=\left(\sum_{n=1}^{N} A_{n} e^{-j \xi_{n}} e^{j k_{0} \sin \theta z_{n}}\right) \times S(\theta) .
$$

We recognize in (13) the expression of the product of the array factor of a one-dimensional antenna array fed in series by the radiation pattern of a single emitter [10]. In (13), the amplitude of each emitter can be tapered.

If the length of a UC is very small compared to the guided wavelength, it is easy to show that $S(\theta)$ becomes independent of $\theta$, each radiator thus becomes an isotropic one.

Also, in the special case of a non-tapered quasi-uniform LWA with very small UC, (13) takes the exact same form as the equation presented in [7].

The directivity of a tapered quasi-uniform LWA is calculated the same way as it is done for classical arrays $[11,12]$ from the radiation pattern, $R_{s}(\theta)$. In the case of small $\mathrm{UC}$ (i.e. isotropic radiators), it is:

$$
D_{s}(\theta)=\frac{\left|R_{s}(\theta)\right|^{2}}{\sum_{n=1}^{N} \sum_{q=1}^{N} A_{n} A_{q} e^{-j\left(\xi_{n}-\xi_{q}\right)} \frac{\sin \left(k_{0}\left(z_{n}-z_{q}\right)\right)}{k_{0}\left(z_{n}-z_{q}\right)}} .
$$

Although the derivation of (12) only considers radiation plane of the elevation-angle $(\theta)$, the fields do exist in the transverse dimension of the LWA aperture ( $y$-axis in Fig. 1). Those fields do contribute to the radiation pattern in the other plane and also to the associated whole directivity.

\section{B. Limits and Potential Applicability of the GAFA}

Regarding the derivation of (13), different approximations that have been made can limit the accuracy of the approach. First, equation (4) shows that the continuous leaky-wave tapered functions $\alpha(z)$ and $\beta(z)$ are sampled. The effect of the sampling can be evaluated directly from the equivalent array factor obtained from (13). If we consider a LWA having a uniform spacing $d$ between the UCs and also identical UCs, the sampling theorem [13, 14] can be applied. Thus, the Nyquist sampling criterion has to be verified at the top surface of the LWA where the radiation begins. Condition is then: $d / \lambda_{g} \leq 1 / 2$. The condition has to be respected also in the far-field: $\left(d / \lambda_{0}<1 / 2\right)$ to ensure that no grating lobes will appear in the visible region [13] and therefore that the obtained radiation pattern corresponds to the one of the continuous illumination distribution $\widetilde{M}(z)$. We can note that due to the radiation condition: $\beta<k_{0}$, the first condition will be respected if the second one is. For quasi-uniform LWAs, the condition is usually satisfied due to the small value of the periodicity. If the Nyquist condition is not respected, the discretized expression (13) takes into account the influence of the grating lobes. The radiation pattern obtained with (13) can be compared to the desired one (continuous distribution) to quickly assess their effect.

It is interesting to note that the approach can be applied to uniform tapered LWAs as the ones studied in $[8,15,16]$. In this case however, there is no UC but the structures are generally discretized for numerical computation. So, any sampling distance that satisfies the Nyquist sampling criterion can be chosen. Furthermore, it is possible to show that the discrete expression (13) takes the exact same form as the continuous expression (1) when the spatial sampling decreases toward zero. The demonstration is given in the appendix.

The approach is also applicable with some restrictions to the analysis of periodic LWAs operating with higher-order Floquet-modes for which $d / \lambda_{0}$ is normally equal to $1 / 2$ for broadside radiation. If multiple space harmonics exist in the structure, each of them can be described separately with (12). However, LWAs are generally optimized for only one harmonic and generally, it must be ensured that all modes except the desired one are non-propagating in the $x$-direction $[16,17]$.

The GAFA method can be applied to tapered LWAs in which both the $\alpha(z)$ and $\beta(z)$ functions are simultaneously varied, since the formulation supports both variations. Indeed, although the majority of the works done when tapering LWAs consists in tapering the amplitude of the aperture distribution while maintaining the phase constant along the antenna to get a specific radiation pattern, some recent works showed that it is interesting to taper the phase $[9,18,19]$. In the case of nearfield focusing antennas, it is interesting to note that the sampling criterion has to be modified compared to far-field applications [14].

Finally, we can also note that the GAFA method can describe structures having non-uniform spacing. This approach used in combination with design procedures for unequally spaced array [20] could lead to new kinds of designs. However, special attention should be paid to the fact that, for LWAs, the UCs are fed through the propagation of the wave inside the waveguide. The phase and magnitude function of the $n^{\text {th }}$ cell thus depends on the previous cells as indicated by the sums of (12). Contrarily to classical antenna arrays, the phase and magnitude functions can thus not be set completely independently from one cell to another for LWAs. 


\section{DESIGN OF DISCRETE TAPERED LWAS}

The design procedure of a tapered uniform LWAs is well established [2] and several examples of implementation can be found in literature $[8,9]$. It includes three steps represented schematically on Fig. 3.

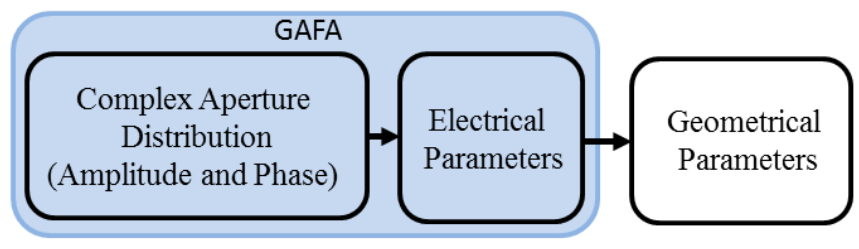

Fig. 3 Design procedure of tapered uniform LWA.

As indicated on Fig. 3, the GAFA is an efficient analysis tool when the geometrical dispersion of the complex propagation constant is already known. However, it does not provide any help for the last step shown in Fig. 3 which is generally considered as the most difficult part of the design. It is also important to note that the accuracy of the GAFA relies directly on the accurate knowledge of the complex propagation constant of the leaky line.

The first step of the procedure is the choice of the tapering function for the aperture illumination amplitude. For that, the use of any classical technique for tapered antenna arrays or uniform LWAs is possible. For instance, optimal solutions in terms of sidelobe level can be employed [10] as well as beam shaping techniques [8]. The distribution functions may be discrete or continuous. In the latter case, the Nyquist condition has to be verified so that the discretization errors can be considered negligible [13].

The second step is the computation of the attenuation constant for each UC of the LWA. Assuming that the phase constant $\beta$ does not change with $z$ [2], the attenuation is calculated with the following formula:

$$
\alpha_{n}=\frac{1}{2} \frac{\left|M_{n}^{0}\right|^{2}}{\frac{1}{\eta} \sum_{i=1}^{N} d_{i}\left|M_{i}^{0}\right|^{2}-\sum_{i=1}^{n} d_{i}\left|M_{i}\right|^{2}},
$$

where $\eta$ is the efficiency of the antenna. The summations appear naturally when describing $\alpha$ with (4). Equation (15) is the discretized version of the equation used for tapered uniform LWAs [2].

Concerning the phase constant, a condition is found from (13). Indeed, to obtain the constructive interference of all the individual emitters in a specific direction $\theta_{0}$, the phase terms of each UC have to be equal to an integer multiple of $2 \pi$. This condition is represented schematically Fig 4 . This leads to a classical formula for phase arrays:

$$
\beta_{n}=\frac{2 \pi m}{d_{n}}+k_{0} \sin \theta_{0},
$$

where $m$ is an integer. If the Nyquist condition is satisfied, the radiation condition $\beta<k_{0}$ is imposing to the value of $m$ to be zero. The same relation than the one for continuous structure is thus found between the phase constant $\beta$ and the main beam angle $\theta_{0}$. As commonly found for fast wave structures, it does not depend on the distance between two consecutive cells.

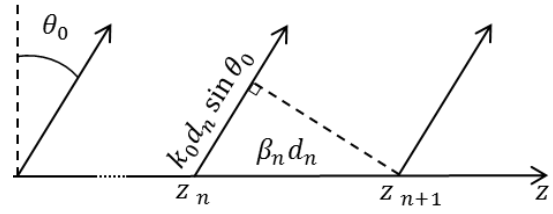

Fig. 4 Path difference between two consecutive unit cells.

A distribution with non-constant values of $\beta$ can lead to phase aberrations compared to the desired radiation pattern. Examples of phase aberrations can be found in $[15,21]$ and their effect are discussed in [22]. The GAFA is a quick method to evaluate phase aberration effects.

The third step of the design procedure is the computation of the geometrical parameters of each UC as a function of $\alpha_{n}$ and $\beta_{n}$. Analytical equations to do that depend on the nature of the LWA. Such relations exist, for example, for Holey and Honey LWAs $[5,6]$. Generally, the analytical equations are valid for non-tapered LWAs, i.e. with constant values of $\alpha$ and $\beta$. Nevertheless, if we consider the single UC $n$ of a tapered LWA to be strictly identical to a UC of a non-tapered LWA with $\alpha_{n}$ and $\beta_{n}$, the transposition is direct.

Couplings exist between adjacent UC of non-uniform LWAs and analytical models for non-tapered LWAs take them into account. In the case of tapered non-uniform LWAs, adjacent UC are different and, strictly speaking, the couplings are not perfectly modeled. However, if the geometry of the UCs is varying in a smooth manner, those coupling can reasonably be assumed to be close to the ones of a non-tapered LWA having constant $\alpha=\alpha_{n}$ and $\beta=\beta_{n}$.

Often, analytical relations that relate the geometrical parameters to the electrical parameters $\alpha$ and $\beta$ of the line are not known. When this is the case, the structure is generally represented by a transverse equivalent network and the geometrical dispersion of $\alpha$ and $\beta$ of the leaky-mode is determined numerically $[8,9]$.

A classical and efficient procedure to fully optimize the radiation pattern of LWAs is to control simultaneously $\alpha(z)$ and $\beta(z)$ along the line thanks to the variation of two different parameters. One geometrical parameter mainly controls the level of leakage, $\alpha(z)$, while the other parameter is maintaining constant $\beta(z)$. Examples of such designs are given in $[8,9,17,22]$.

Finally, let's mention that structures like CRLH LWAs are particularly sensitive to couplings effects. In this case, even for smooth variations of the UC geometry, the values of $\alpha$ and $\beta$ are showing important deviations from their equivalent uniform infinite line counterparts and are not precise enough for a direct design [23]. A more complex design procedure based on numerical methods is then required [24].

\section{NUMERICAL VALIDATION OF SOME PROPOSED TAPERED STRUCTURES}

To validate the GAFA approach for tapered non-uniform LWAs, the design procedure was tested with the Honey LWA and the Holey LWA. In this section, the results are compared with full-wave simulations (CST microwave studio).

\section{A. First Example with the Honey Antenna}

The first design corresponds to the Honey Antenna tapered 
with a Taylor distribution. The Taylor distribution yields decaying minor lobes and an optimal beamwidth for specified sidelobe level. The distribution is [10]:

$$
|\widetilde{M}(z)|=J_{0}\left(j \pi B \sqrt{1-(2 z / L)^{2}}\right)
$$

for $0 \leq z \leq L$ and 0 everywhere else. $J_{0}($.$) is the Bessel$ function of the first kind and $B$ is a constant which controls the sidelobe level. For sidelobe level of $-20 \mathrm{~dB}$ and $-40 \mathrm{~dB}, B$ shall take the values 0.739 and 1.742 respectively. For the current design, the objective is to reduce the sidelobe level to $-20 \mathrm{~dB}$.

For the Honey LWA, several parameters can be used to tune the $\alpha_{n}$ to the desired values, but the most sensitive one is the gap between the metallic strips, $p_{n}$, see Fig. 5. The variations of $\alpha$ and $\beta$ as a function of $p$ are represented in Fig. 6. They are computed from the analytical equations found in [5]. Those equations where derived from an equivalent network analysis and are accurate as long as the electric field is inductive, which is the case for small spacing of the strips compared to the wavelength. They take into account the couplings between adjacent inductive strips. Although the fact that for tapered structures the spacing is varying, the spacing is varied smoothly every four or five strips depending on the position along the line. As the variation is smooth, it is assumed that in a constant section (4 or 5 strips), the propagation constant is close to the one of an infinite line with the same geometry. The values of the $\beta_{n}$ are also computed from analytical equations found in [5] once the $p_{n}$ are known. In the present case, the ratio between the maximum spacing and the wavelength in free space is $d / \lambda_{0}=0.23$. The theoretical efficiency calculated form the distribution of $\alpha$ and the length $L$ is $90 \%$.

The designed antenna is illustrated in Fig. 5 and its parameters are given in Table 1. In the present case, the guide is filled with air. For the full-wave simulation, metallic walls are present on the edges to emulate strips of infinite length. The feed is done with waveguide ports placed sufficiently close to the structure to produce a wave polarized in the horizontal plane which propagates between the bottom and top planes.

The radiations patterns computed with the GAFA method and simulated are illustrated in Fig. 7 and the antenna characteristics are given Table 2. Both results are in good agreement up to $-30 \mathrm{~dB}$ where level differences appear. Positions of the lobes are in good agreement from $-45^{\circ}$ up to $75^{\circ}$. As only one parameter was used for the taperization, no control of $\beta$ was possible creating phase aberrations. The effect of the later are easily evaluated by comparing the GAFA calculations with a varying $\beta$ (dotted blue line in Fig. 7 ) to the ones obtained with a constant value of $\beta$ (dotted grey line in Fig. 7). It is worth noting that Honey did propose a tapered LWA in its original work [5] where the height $a$ of the guide is varied resulting in a curved form of the top conducting surface in order to maintain $\beta$ constant along the structure. He obtained excellent results.

\section{B. Second Example with the Holey Antenna}

The design of Holey antenna has been performed with cosine and Taylor illumination distributions [10]. A continuous

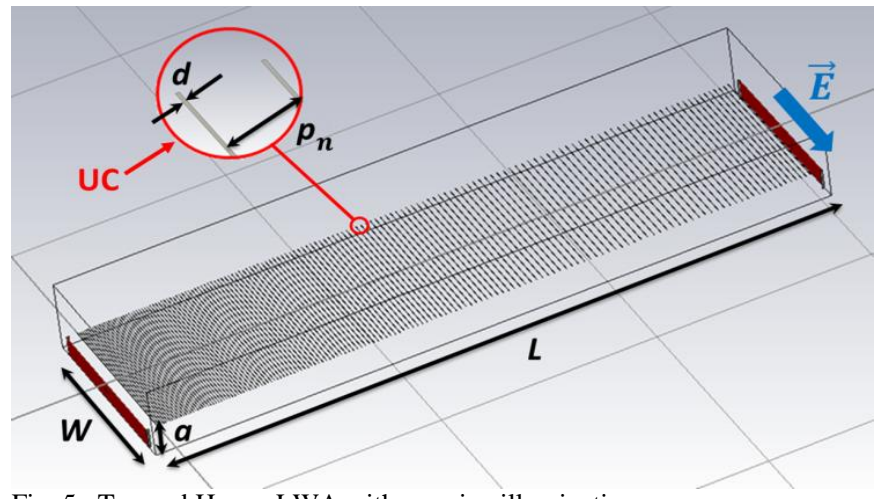

Fig. 5 Tapered Honey LWA with a cosine illumination.

TABle 1 Dimensions of THe Honey LWA

\begin{tabular}{|c|c||c|c|}
\hline \hline Parameter & Value & $\begin{array}{c}\text { Characteristic } \\
\text { quantity }\end{array}$ & Value \\
\hline$a$ & $20.0 \mathrm{~cm}$ & Working frequency, $f_{0}$ & $11.42 \mathrm{GHz}$ \\
\hline$d$ & $0.254 \mathrm{~mm}$ & Pointing angle, $\theta_{0}$ & $54^{\circ}$ \\
\hline$L$ & $609.6 \mathrm{~mm}$ & Number of UC, $N$ & 151 \\
\hline$W$ & $220 \mathrm{~mm}$ & Strip spacing, $p_{n}$ & $1.94-6.05 \mathrm{~mm}$ \\
\hline \hline
\end{tabular}

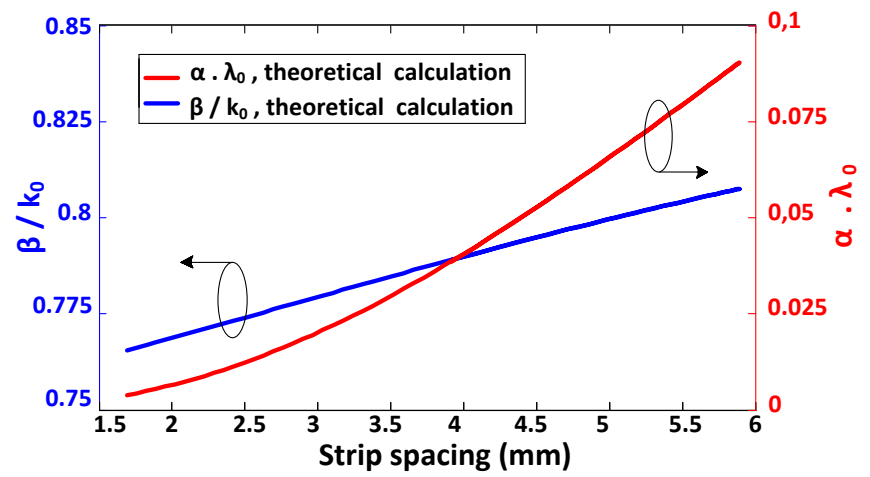

Fig. 6 Normalized complex propagation constants (phase constant and attenuation constant) of a Honey LWA computed with the analytical equations of [5].

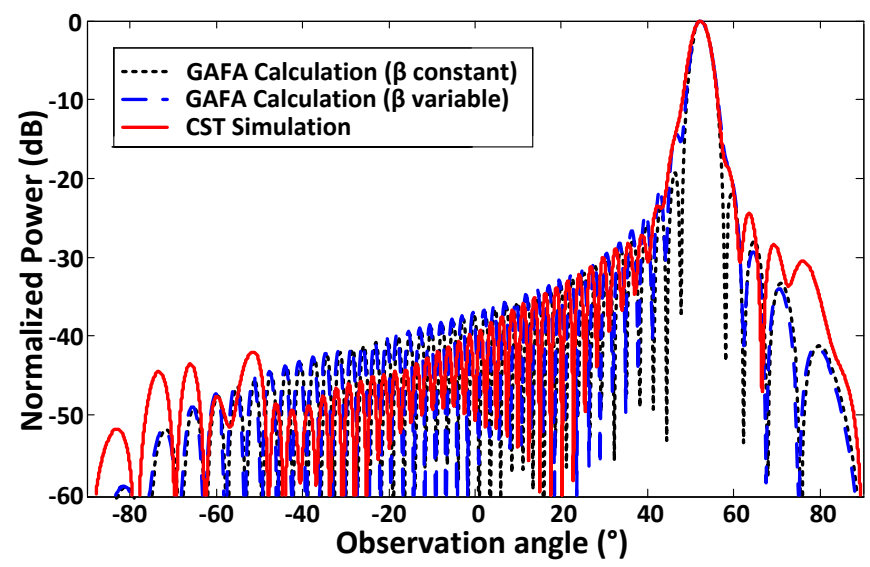

Fig. 7 Radiation patterns of a tapered Honey LWA computed with the GAFA and simulated. Taylor distribution.

cosine distribution provides theoretical sidelobe level of $-23.2^{\circ} \mathrm{dB}$. Its equation is:

$$
|\widetilde{M}(z)|=\sin (\pi z / L) \text {. }
$$


In the present case, the Taylor distribution is determined to reach sidelobe level of $-40 \mathrm{~dB}$ (see previous section).

Antennas like the one shown in Fig. 8 with the parameters given in Table 3 were designed. The diameters of the holes were computed with the help of the analytical equations given in [6]:

$$
\begin{gathered}
\alpha \lambda=\frac{\lambda \lambda_{g 0} R^{\prime}}{2 a^{2}}, \\
\frac{\beta}{k_{0}}=\frac{\lambda}{\lambda_{g}}=\frac{\lambda}{\lambda_{g 0}}\left(1+\frac{\lambda_{g 0}{ }^{2} X^{\prime}}{4 \pi a^{2}}\right), \\
\lambda_{g 0}=\frac{\lambda}{\sqrt{1-(\lambda / 2 a)^{2}}},
\end{gathered}
$$

with:

$$
\begin{array}{cc}
R^{\prime}=\frac{G^{\prime}}{G^{\prime 2}+B^{\prime 2}}, & X^{\prime}=\frac{B^{\prime}}{G^{\prime 2}+B^{\prime 2}}, \\
G^{\prime}=\frac{\pi b}{2 a}, & B^{\prime}=\frac{6 a b p}{\pi c^{3}} .
\end{array}
$$

The diameter of each hole, $c_{n}$, is the only parameter which can be easily varied for the Holey antenna. Thus, it is not possible to taper $\alpha$ while maintaining $\beta$ constant. However holes do not disturb much the electric field and the variations of the $c_{n}$ have a limited impact on $\beta$. In the range of values used for the $c_{n}$ (15 to $35 \mathrm{~mm}$ for the cosine distribution and 0.9 to $29.5 \mathrm{~mm}$ for the Taylor one), the variation of $\beta$ is $9 \%$, see Fig. 9.

The validity range of (19) and (20) was checked for the antenna design. For that purpose, different uniform LWAs having the same length $L$ and the same periodicity $p$ as the final tapered LWA where simulated with a commercial software (CST Microwave Studio). The diameters of the holes, $c$, were varied from one simulation to another and the corresponding $S$-parameters where obtained. Classical

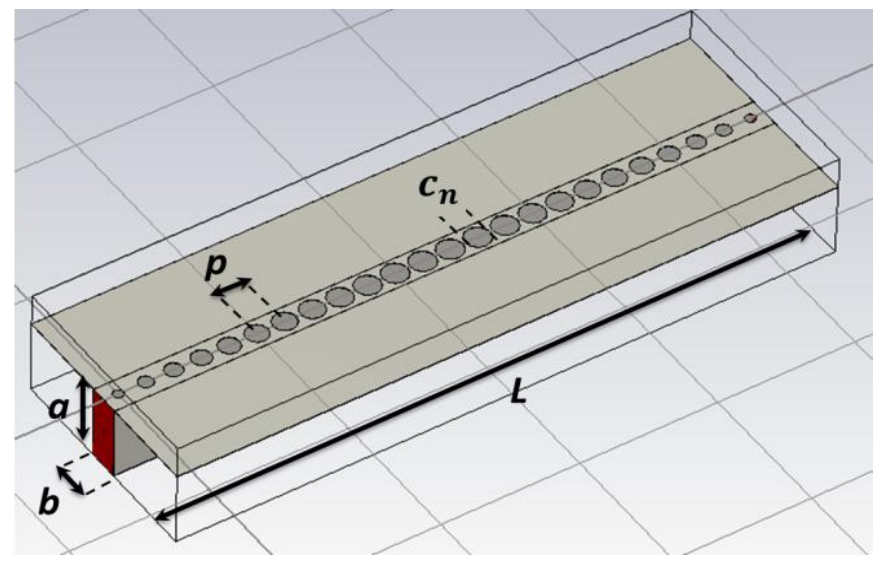

Fig. 8 Tapered Holey LWA with a cosine illumination.

TABle 3 DimensiOnS OF THE HOLEy LWA

\begin{tabular}{|c|c||c|c|}
\hline \hline Parameter & Value & $\begin{array}{c}\text { Characteristic } \\
\text { variable }\end{array}$ & Value \\
\hline$a$ & $12.0 \mathrm{~cm}$ & Cutoff frequency, $f_{c}$ & $1.25 \mathrm{GHz}$ \\
\hline$b$ & $4.5 \mathrm{~cm}$ & Working frequency, $f_{0}$ & $1.5 \mathrm{GHz}$ \\
\hline$p$ & $4.0 \mathrm{~cm}$ & Pointing angle, $\theta_{0}$ & $\begin{array}{c}33^{\circ} \text { cosine } \\
31^{\circ} \text { Taylor }\end{array}$ \\
\hline$L$ & $100 \mathrm{~cm}$ & Number of holes, $N$ & 24 \\
\hline \hline
\end{tabular}

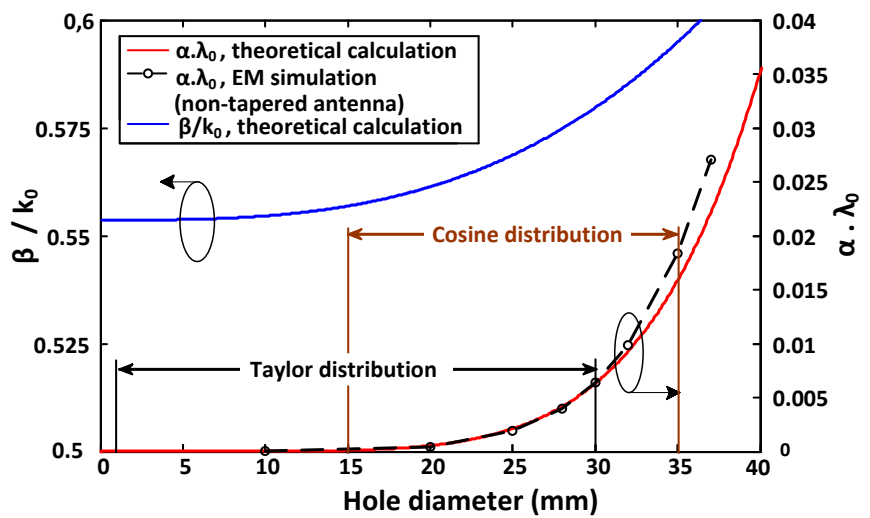

Fig. 9 Variations of the normalized phase constant and of the normalized attenuation constant obtained with the analytical equations of [6]. The latter is also compared to the one extracted from electromagnetic simulations.

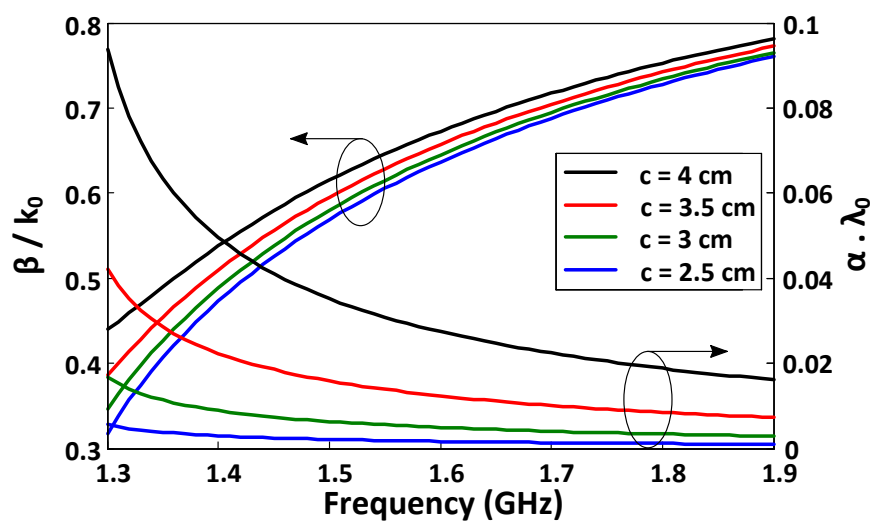

Fig. 10 Variations of the normalized phase constant and of the normalized attenuation constant with respect to the frequency, obtained with the analytical equations of [6].

extraction formulas [7] were used to deduce the values of $\alpha$ associated to each hole diameter. The obtained values are indicated by black circles on Fig. 9 and are compared with the ones given by the analytical equations (19-20). Arbitrarily, differences of $\alpha$-values obtained from both calculations less than $20 \%$ were considered acceptable, see Fig. 9. This gives a range of values for $\alpha$ : $0 \leq \alpha \lambda \leq 0.025$. However, this range limits the efficiency of the antenna to only $15 \%$ with the cosine distribution and to $11 \%$ with the Taylor distribution. Those antennas have thus no real practical interest as such. Those small efficiencies are due to the fact that the obtainable leakage rates are too low for the chosen lengths $\left(L=5 \lambda_{0}=1 \mathrm{~m}\right)$ of the antennas. In order to obtain an efficiency of $90 \%$, the maximum value of $\alpha . \lambda$ should be 0.44 and 0.75 for the Cosine and Taylor distributions respectively. Those values are not realizable in practice for such structure.

We shall however note that the objective of the design is to validate the GAFA approach and that no real application is target here. The length $L$ is thus arbitrarily chosen without considerations for the classical specifications (beamwidth, directivity) in such a way that the computation time of the fullwave simulations was small enough. The normalized illumination distribution is also chosen arbitrarily and the efficiency in (15) is used as an adjustment parameter to obtain realizable values of $\alpha$.

The ratio between the spacing and the wavelength in free space is $p / \lambda_{0}=0.2$ and it thus verifies the Nyquist criterion. 
The evolution of the normalized phase constant and normalized attenuation constant with respect to the frequency has been represented in Fig. 10 for various values of the diameter of the hole.

The radiation patterns simulated with CST and calculated with (13) for both distributions are shown in Fig. 11. LWAs are simulated without transitions and are fed via waveguide ports. The impedance of the ports correspond to the one of the closed (not disturb by the holes) waveguide. The magnitude values of $S_{I I}$ given in Table 2 are the one computed at the ports by CST Microwave studio. The comparison between the curves of Fig. 11 shows a good agreement. The latter is even better when the real values of $\beta$ are used (i.e. the curves " $\beta$ variable"). The variations of $\beta$ are due to the fact that only one parameter is used to control $\alpha$. One advantage of the GAFA method is that it can be used to rapidly make a first estimation of the impact of the fluctuation of $\beta$ on the width of the main lobe. This is done by forcing $\beta$ to a constant value, see Fig. 11 . In the present case, at $-20 \mathrm{~dB}$, the non-controlled fluctuations of $\beta(z)$ creates an increase of the main beam width of $4^{\circ}$ and $8^{\circ}$ respectively for both distributions.

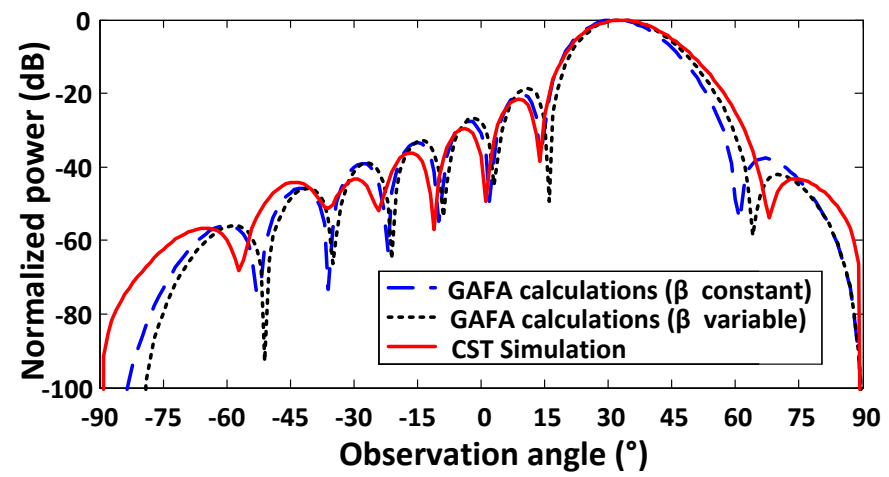

(a)

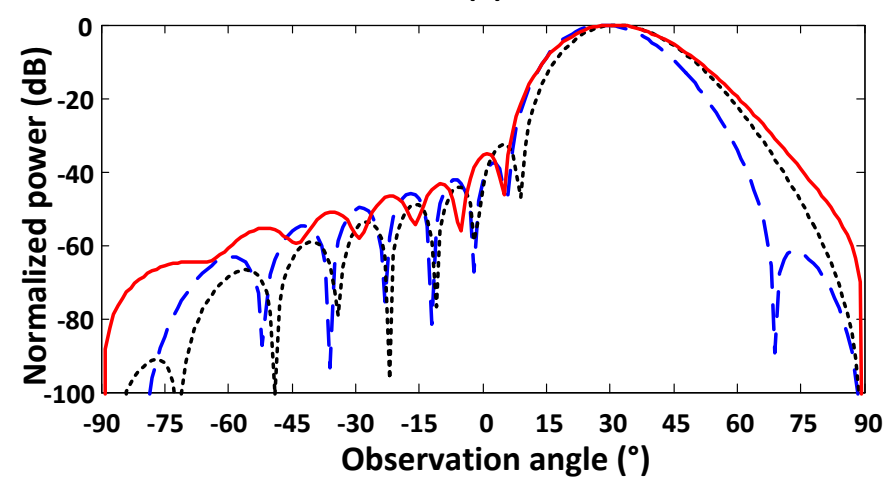

(b)

Fig. 11 Radiation patterns of a tapered 24-cells Holey LWA computed with the generalized array factor approach and simulated. Numerical values are given in Table 3. a) Cosine distribution. b) Taylor distribution.

TABle 2 Simulation Results

\begin{tabular}{|c|c|c|c|c|}
\hline \hline \multicolumn{2}{|c|}{ Antenna } & Honey & \multicolumn{2}{c|}{ Holey } \\
\hline \multicolumn{2}{|c|}{ Distribution } & Taylor & Cosine & Taylor \\
\hline \multirow{2}{*}{ SLL } & Theory & $-20 \mathrm{~dB}$ & $-23.2 \mathrm{~dB}$ & $-40 \mathrm{~dB}$ \\
\cline { 2 - 5 } & Simulation & $-14.1 \mathrm{~dB}$ & $-21.6 \mathrm{~dB}$ & $-34.9 \mathrm{~dB}$ \\
\hline \multicolumn{2}{|c|}{ Directivity } & $27.7 \mathrm{~dB}$ & $13.01 \mathrm{dBi}$ & $12.61 \mathrm{dBi}$ \\
\hline \multicolumn{2}{|c|}{ Adaptation $\left(\mathrm{S}_{11}\right)$} & $-19.88 \mathrm{~dB}$ & $-42.88 \mathrm{~dB}$ & $-55.28 \mathrm{~dB}$ \\
\hline \multicolumn{2}{|c|}{ Main beam angle } & $54^{\circ}$ & $33^{\circ}$ & $31^{\circ}$ \\
\hline \hline
\end{tabular}

\section{MEASUREMENT Results}

An experimental validation of the GAFA method was carried out. A third model of a Holey antenna with a cosine distribution has been realized and measured. The dimensions of the structure have been modified compared to the ones presented in sect IV. B in order to comply with the WR430 standard dimensions operating in the $\mathrm{R}$ band $(1.7-2.6 \mathrm{GHz})$. The working frequency has been chosen at $1.7 \mathrm{GHz}$ to decrease the far-field distance region $(6.38 \mathrm{~m})$ and to improve the efficiency of the antenna. Dimensions and characteristic values of the antenna are given in Table 4 . A picture of the realized antenna is shown in Fig. 12.

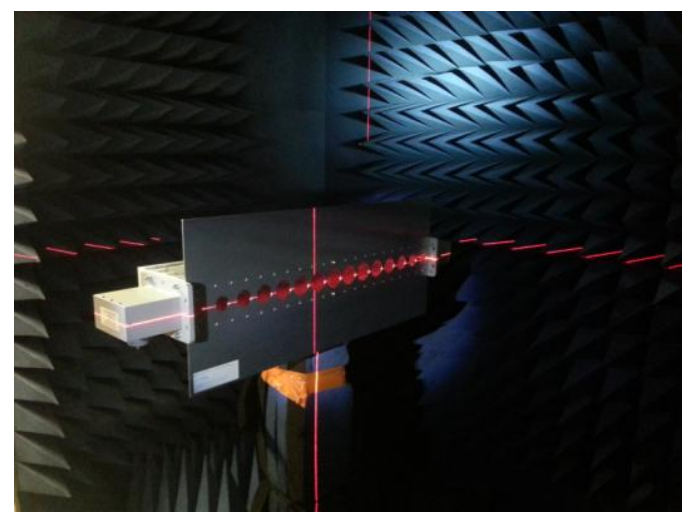

Fig 12. Picture of the Holey LWA used during the measurement in an anechoic chamber.

TABle 4 Dimensions of THE REALIZED Holey LWA (FIG. 12)

\begin{tabular}{|c|c||c|c|}
\hline \hline Parameter & Value & $\begin{array}{c}\text { Characteristic } \\
\text { variable }\end{array}$ & Value \\
\hline$a$ & $10.92 \mathrm{~cm}$ & Cutoff frequency, $f_{c}$ & $1.37 \mathrm{GHz}$ \\
\hline$b$ & $5.46 \mathrm{~cm}$ & Working frequency, $f_{0}$ & $1.7 \mathrm{GHz}$ \\
\hline$p$ & $5.0 \mathrm{~cm}$ & Pointing angle, $\theta_{0}$ & $39^{\circ}$ \\
\hline$L$ & $75 \mathrm{~cm}$ & Number of holes, $N$ & 15 \\
\hline \hline
\end{tabular}

The transitions used to feed the LWA are commercial ones (Vector Telecom VT22WCASKPA). They give a maximum VSWR of 1.5 in the frequency band 1.7-2.6 GHz. One of the terminations was connected to an adapted load in order to avoid reflection of the remaining power at the end of the waveguide. Because no information was available concerning the geometrical dimensions of the transitions, it was not possible to include the transitions in the full-wave simulations of the LWA.

The measured magnitude of the $\mathrm{S}$ parameters is represented on Fig. 13. At the working frequency, the measured magnitude of the $S_{11}$ parameter of the antenna together with the two transitions is $-33 \mathrm{~dB}$ and the magnitude of the $S_{21}$ parameter is equal to $-0.79 \mathrm{~dB}$. The measured efficiency given in Table 5, is evaluated from the $S$ parameters as $\eta=1-\left|S_{11}\right|^{2}-\left|S_{12}\right|^{2}$.

The comparison of the radiation patterns calculated with (12), simulated with CST and measured is given in Fig. 14. The measurement, simulation and theory are showing a fair agreement in the main lobe region: $-10^{\circ}<\theta<90^{\circ}$. A shift of $1.5^{\circ}$ of the main beam angle, $\theta_{0}$, is observed between theory and measurements. The absence of the first null can probably be attributed to phase aberration. A sidelobe level of $-21.2 \mathrm{~dB}$ has been measured which is in good agreement with the theoretical value of $-21.6 \mathrm{~dB}$. In the region $-90^{\circ}<\theta<-10^{\circ}$, the CST simulation and the measurement are in acceptable 


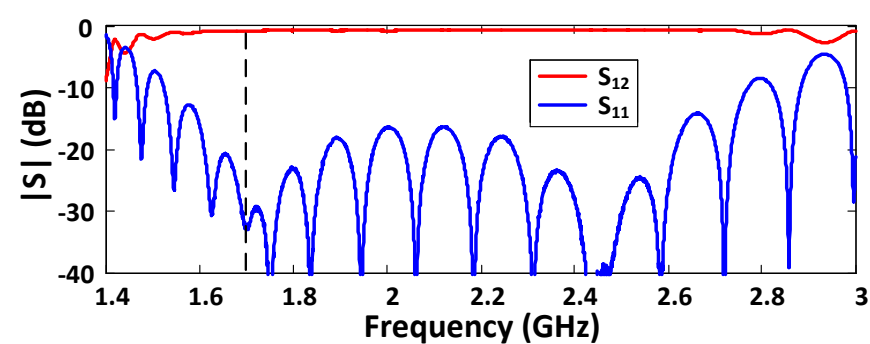

Fig 13. Measured magnitude of the S parameter of the realized Holey LWA

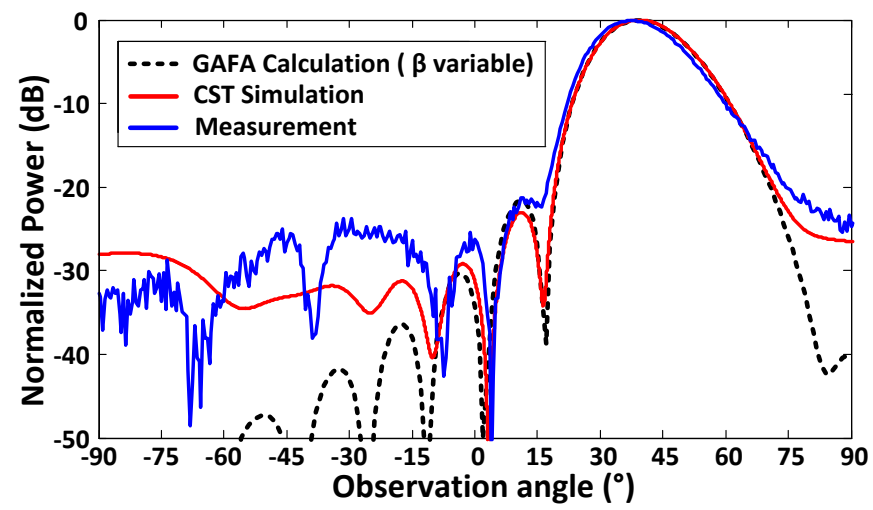

Fig 14. Radiation pattern of a Holey antenna calculated with the GAFA method, simulated and measured. Cosine distribution.

agreement, but a clear discrepancy is observed between them and the theoretical prediction (GAFA calculation). The ripples in this region are showing a maximum value of $-23.75 \mathrm{~dB}$ for measurement and $-28 \mathrm{~dB}$ for simulation.

Contrarily to the design of section IV. B, the equations (1920) have been used for present design without any prior verification of their validity range. As it can be seen on Fig. 9, this can lead to important inaccuracies in the assessment of $\alpha$ and $\beta$. This can explain the discrepancy observed between the radiation pattern obtained in theory and measurement. When the propagation parameters are estimated with sufficient accuracy like in the section IV. B, the results are showing a better agreement for the entire visible region $\left(-90^{\circ}<\theta<90^{\circ}\right)$ (see Fig. 11).

The value of the directivity at $\theta_{0}$ has been calculated with (13) for the case of an isotropic emitter, see Table 5. The simulated value shows an augmentation of $0.7 \mathrm{dBi}$ compared to the theoretical one. This is probably due to the fact that only the elevation-angle $(\theta)$ was considered in (14), The fields in the transverse dimension of the LWA aperture are not perfectly isotropic and then contributes to the whole directivity. The measured value is a bit lower than simulation

TABLE 5 CHARACTERISTICS COMPARISON BETWEEN THEORY, SIMULATI
\begin{tabular}{|c||c|c|c|}
\hline \hline & Theory & Simulation & Measurement \\
\hline \hline SLL (dB) & -21.6 & -23 & -21.2 \\
\hline $\begin{array}{c}\text { Directivity } \\
\text { (dBi) }\end{array}$ & 10.4 & 11.3 & 10.7 \\
\hline Efficiency & 0.15 & 0.13 & 0.09 \\
\hline $\begin{array}{c}\text { Main beam } \\
\text { angle }\end{array}$ & $-39.5^{\circ}$ & $-39.6^{\circ}$ & $-38^{\circ}$ \\
\hline \hline
\end{tabular}

$(-0.5 \mathrm{dBi})$ but within the measurement uncertainty range $( \pm 1.5$ $\mathrm{dBi}$ ). Let's us note that for the calculation of the measured directivity value, the efficiency obtained by simulation has been used.

Measurements for different working frequencies (from $1.5 \mathrm{GHz}$ to $1.9 \mathrm{GHz}$ ) have been realized and have shown that the sidelobe level sensitivity with respect to frequency is $+0.4 \mathrm{~dB} / 100 \mathrm{MHz}$ in the vicinity of the working frequency. The main beam direction is shifted from $+15^{\circ}$ for the $400 \mathrm{MHz}$ frequency variation. Those results are in good agreement with (16).

\section{CONCLUSION}

A generalization of the array factor approach was demonstrated and applied to tapered non-uniform LWAs. The demonstration is based on the Fourier transform of the illumination amplitude of the LWA. The concept was tested and found very efficient with the Holey and the Honey LWA for which analytical expressions that relate the parameters $\alpha$ and $\beta$ to the geometrical dimensions of the antenna do exist. The simulations have shown a good agreement with theory for both cosine and Taylor distributions. An experimental validation has been done for a Holey antenna. Measurements are in acceptable agreement with the theory and the numerical simulations. The Generalized Array Factor is certainly a welladapted technique to design tapered discrete LWAs. It could be used with benefit for the design of tapered CRLH LWAs.

\section{APPENDIX}

Annexe A: Equivalence between the discrete and the continuous radiation pattern for infinitely small spacing.

Mathematically, in (12)-(13) the sampling in the exponentials, that will be denoted by $p$, can be distinguished from the one of the sum: $d$. In this case, $R_{S}(\theta)$ is expressed as:

$$
\begin{aligned}
R_{S}(\theta) & =v_{p}(d)=\sum_{n=1}^{N} n A d \sqrt{\alpha(n d)} \exp \left(-\sum_{i=1}^{M-1} p \alpha(i p)\right) \\
& \times \exp \left(-j \sum_{i=1}^{M-1} p \beta(i p)\right) e^{j n d k_{0} \sin \theta} \cdot S_{M}{ }^{p}(\theta),
\end{aligned}
$$

with $M$ defined like: $(M-1) p \leq n d<M p$ and where $S_{M}{ }^{p}(\theta)$ denotes:

$$
\begin{aligned}
& S_{M}{ }^{p}(\theta)=\int_{0}^{1} \sqrt{a(t)} \exp \left(-p . \alpha(M p) \int_{0}^{t} a(\tau) d \tau\right) \\
& \quad \times \exp \left(-j p . \beta(M p) \int_{0}^{t} b(\tau) d \tau\right) e^{j k_{0} \sin \theta t p} d t .
\end{aligned}
$$

When $p \rightarrow 0, M$ becomes infinite, and we have:

$$
\begin{gathered}
\lim _{p \rightarrow 0}(M-1) p=n d, \\
\lim _{p \rightarrow 0} S_{M}{ }^{p}(\theta)=1 .
\end{gathered}
$$

As the functions $\alpha($.$) and \beta($.$) considered in (21) are$ generally bounded and defined on a finite interval, the double 
limit theorem can be applied which leads to the final result:

$$
\begin{aligned}
& \lim _{d \rightarrow 0} R_{S}(\theta)=\lim _{d \rightarrow 0} v_{d}(d)=\lim _{d \rightarrow 0 p \rightarrow 0} \lim _{p}(d) \\
& =\lim _{d \rightarrow 0} \sum_{n=1}^{N} n A d \sqrt{\alpha(n d)} \exp \left(-\int_{0}^{n d} \alpha(\tau) d \tau\right) \\
& \quad \times \exp \left(-j \int_{0}^{n d} \beta(\tau) d \tau\right) e^{j n d k_{0} \sin \theta} \\
& =R(\theta) .
\end{aligned}
$$

\section{REFERENCES}

[1] D. R. Jackson, "Recent Advances in Leaky-Wave Antennas," in 2013 International Symposium on Electromagnetic Theory (EMTS) - URSI Comission B, Hiroshima, Japan, 2013, pp. 1-4.

[2] A. A. Oliner and D. R. Jackson, "Leaky-Wave Antennas," in Antenna engineering handbook, J. L. Volakis, Ed., 4th ed: McGraw-Hill, 2007.

[3] D. R. Jackson, C. Caloz, and T. Itoh, "Leaky-Wave Antennas," Proceedings of the IEEE, vol. 100, pp. 2194-2206, 2012.

[4] C. Caloz and T. Itoh, "TL Theory of MTMs," in Electromagnetic Metamaterials: Transmission Line Theory and Microwave Applications, ed: John Wiley \& Sons, Ch. 3, pp. 106-113, 2005.

[5] R. C. Honey, "A flush-mounted leaky-wave antenna with predictable patterns," IRE Transactions on Antennas and Propagation, vol. 7, pp. 320-329, 1959.

[6] L. Goldstone and A. A. Oliner, "Leaky-wave antennas I: Rectangular waveguides," IRE Transactions on Antennas and Propagation, vol. 7, pp. 307-319, 1959.

[7] C. Caloz and T. Itoh, "Array factor approach of leaky-wave antennas and application to 1-D/2-D composite right/left-handed (CRLH) structures," Microwave and Wireless Components Letters, IEEE, vol. 14, pp. 274-276, 2004.

[8] J. L. Gomez-Tornero, A. J. Martinez-Ros, and R. Verdu-Monedero, "FFT Synthesis of Radiation Patterns With Wide Nulls Using Tapered Leaky-Wave Antennas," Antennas and Wireless Propagation Letters, IEEE, vol. 9, pp. 518-521, 2010.

[9] A. J. Martinez-Ros, J. L. Gomez-Tornero, and G. Goussetis, "Holographic Pattern Synthesis With Modulated Substrate Integrated Waveguide Line-Source Leaky-Wave Antennas," Antennas and Propagation, IEEE Transactions on, vol. 61, pp. 3466-3474, 2013.

[10] C. A. Balanis, Antenna Theory: Analysis and Design., Third ed. Hoboken, N.J.: Wiley-Interscience, 2005.

[11] W. L. Stutzman and G. AA. Thiele, Antenna Theory and Design., $2^{\text {nd }}$ ed. New York: Wiley, 1998.

[12] Casares-Miranda, F.P.; Camacho-Penalosa, C.; Caloz, C., "High-gain active composite right/left-handed leaky-wave antenna," IEEE Trans. on Ant. and Prop., vol.54, no.8, pp.2292,2300, Aug. 2006.

[13] A. K. Bhattacharyya "Phased Array Fundamentals: Pattern Analysis and Synthesis," in Phased Array Antennas: Floquet Analysis, Synthesis, BFNs, and Active Array Systems, $1^{\text {st }}$ ed., John Wiley, 2006, ch. 1, sec. 1.6, pp. 53-56.

[14] A.D. Yaghjian, "An overview of near-field antenna measurements," IEEE Trans. on Ant. and Prop., vol. 34, no. 1, pp. 30-45, Jan. 1986.

[15] J. L. Gomez-Tornero, A. T. Martinez, D. C. Rebenaque, M. Gugliemi, and A. Alvarez-Melcon, "Design of Tapered Leaky-Wave Antennas in Hybrid Waveguide-Planar Technology for Millimeter Waveband Applications," IEEE Trans. on Ant. and Prop., vol. 53, pp. 2563-2577, 2005.

[16] Y. J. Cheng, W. Hong, K.Wu, and Y. Fan, "Millimeter-wave substrate integrated waveguide long slot leaky-wave antennas and twodimensional multibeam applications," IEEE Trans. on Ant. and Prop., vol. 59, no. 1, pp. 40-47, Jan. 2011.

[17] J.L. Gómez, F.D. Quesada and A.A. Melcón, "Analysis and Design of Periodic Leaky-Wave Antennas for the Millimeter Waveband in Hybrid Waveguide-Planar Technology", IEEE Trans. on Ant. and Prop., Vol.53, No.9, pp. 2834-2842, Sep. 2005.

[18] J.-L. Gomez-Tornero, "Unusual tapering of leaky-wave radiators and their applications," in Proc. 5th Eur. Conf. Ant. Prop. (EUCAP), Rome, Italy, Apr. 2011, pp. 821-824

[19] A. J. Martinez-Ros and J. L. Gomez-Tornero, "Free space near field focusing from an array of sinusoidally modulated half-mode LWAs," in
2013 IEEE Ant. and Prop. Soc. Int. Symp. (APSURSI), Jul. 2013, pp. $1568-1569$.

[20] A. Ishimaru, "Unequally Spaced Arrays Based on the Poisson Sum Formula," IEEE Trans. on Ant. and Prop., vol. 62, no. 4, pp. 15491554, Apr. 2014

[21] M. García-Vigueras, J.L. Gómez-Tornero, G. Goussetis, A. R. Wiley, and Y. Jay Guo, "Efficient Synthesis of 1D Fabry-Perot Antennas with Low Sidelobe Levels," IEEE Ant. and Wireless Prop. Lett., vol.11, pp. 869 - 872, August 2012.

[22] S. Silver, Microwave Antenna Theory and Design, mcgraw-Hill Book Co., New York, ny, Ch. 6

[23] O. Rance, P. Lemaitre-Auger, R. Siragusa and E. Perret, "Non-uniform description of short CRLH Leaky Wave Antenna with the Generalized Array Factor Approach," 44th European Microwave Conference (EuMC),pp. 247-250, 6-9 Oct. 2014

[24] R. Siragusa, E. Perret, P. Lemaitre-Auger, H. Van Nguyen, S. Tedjini, and C. Caloz, "A Tapered CRLH Interdigital/Stub Leaky-Wave Antenna With Minimized Sidelobe Levels," Antennas and Wireless Propagation Letters, IEEE, vol. 11, pp. 1214-1217, 2012.

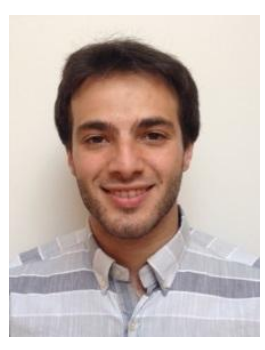

Olivier Rance (S'15) is a $\mathrm{PhD}$ student working under the supervision of Dr. Etienne Perret. He received his Master's degree in Electronic Engineering from the Institute National Polytechnique de Grenoble (Grenoble-INP), and joined the LCIS in 2012. His main research interests are leaky-wave antennas and chipless RFID.

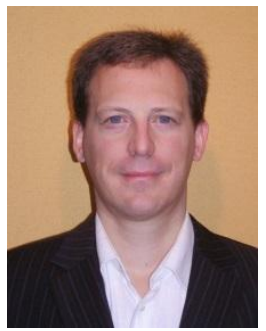

Pierre Lemaitre-Auger received the B. Ing. and the M. Sc. A. degrees from the École Polytechnique de Montréal, Canada, in 1992 and 1994 respectively in physical engineering. He obtained his doctor degree in Optoelectronics and Microwave from the Institut National Polytechnique de Grenoble, France, in 1998. He joined the engineering school Esisar (Valence, France) of the Institut Polytechnique de Grenoble and the LCIS Laboratory in 1998 as an associate professor He participated to the creation of the Esisar and especially to the elaboration of its physics teaching program. He directed the Physical Department of the Esisar from 1997 to 2006. From 2006 to 2009, he was the Director of Study of that same school. In 2010-2011, he was visiting professor at the École Polytechnique de Montréal in the Poly-Grames Microwave Research Center. Since 2014, he is Head of International Relations of Esisar. His first research topics concerned integrated optics on glass substrates for sensors applications. From 2004 to 2010, he actively participated to the creation of a French startup company specialized in optical sensors. In 2006, he changed his research activities to electromagnetic wave generation and propagation: antennas, array antennas, frequency selective surface and localized waves. He is the author and co-author of more than 80 journal papers, letters, and international conferences. He is co-author of 5 patents. His work on wall-paper blocking WiFi signals won several French awards. 


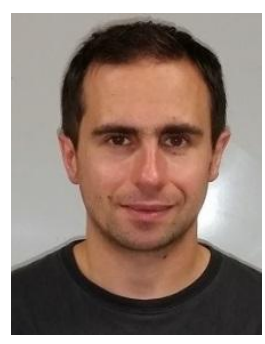

Romain Siragusa was born in Paris, France. He received the engineer degree and the master degree from the Institute National Polytechnique de Grenoble (Grenoble-INP) in 2006 and the Ph.D degree from the same institute at the Laboratoire de Conception et d'Intégration des Systèmes (LCIS) in Valence, France. In 2010/2009, he joined the "commissariat à l'énergie atomique" (CEA) as post doctoral fellow where he studied the high impedance surface for integrated antenna on silicon. Since 2011, he is associate professor at Grenoble-INP Esisar / LCIS. His current scientific interests include leakywave structures and Chipless RFID.

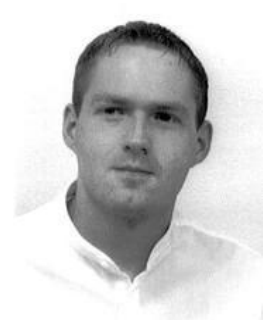

Etienne Perret received the Eng. Dipl. in electrical engineering from the Ecole Nationale Supérieure d'Electronique, d'Electrotechnique, d'Informatique, d'Hydraulique, et des Télécommunications, Toulouse, France, in 2002, and the M.Sc. and Ph.D. degrees from the Toulouse Institute of Technology, France, in 2002 and 2005, respectively, all in electrical engineering. From 2005 to 2006, he held a post-doc position at the Institute of Fundamental Electronics (IEF), Orsay, France. Since September 2006, Dr. Perret is Associate Professor in electrical engineering with the Grenoble Institute of Technology. In October 2014, he was elevated to Junior member of the "Institut Universitaire de France", an institution that distinguishes professors for their research excellence, as evidenced by their international recognition.

$\mathrm{He}$ is authored and co-authored of more than 120 technical conferences, letters and journal papers, and books. He is IEEE senior member and Technical Program Committee member of the IEEE International Conference on RFID. He was keynote speaker and the chairman of several international symposiums. His research activities cover the electromagnetic modeling of passive devices for millimeter and submillimeter-wave applications. His current research interests are in the field of wireless communications, especially radio frequency identification (RFID) and chipless RFID. His interests also involve advanced computer aided design techniques based on the development of an automated co-design synthesis computational approach. He was named one of MIT Technology Review's French Innovator's under 35 in 2013 for his work on chipless RFID. He is also the recipient of the French's Innovative techniques for the environment awards in 2013. 